\title{
Crystal structure of bis(benzamide-2-thiolato) $\left(\mu_{2}\right.$-bis(diphenylphosphino)- ethane)digold(I), $\mathrm{C}_{40} \mathrm{H}_{36} \mathrm{Au}_{2} \mathrm{~N}_{2} \mathrm{O}_{2} \mathbf{P}_{2} \mathrm{~S}_{2}$
}

\author{
D. R. Smyth and E. R. T. Tiekink*.1 \\ The University of Adelaide, Department of Chemistry, Australia 5005
}

Received June 10, 2002 accepted and available on-line August 6, 2002; CCDC-No. 1267/878

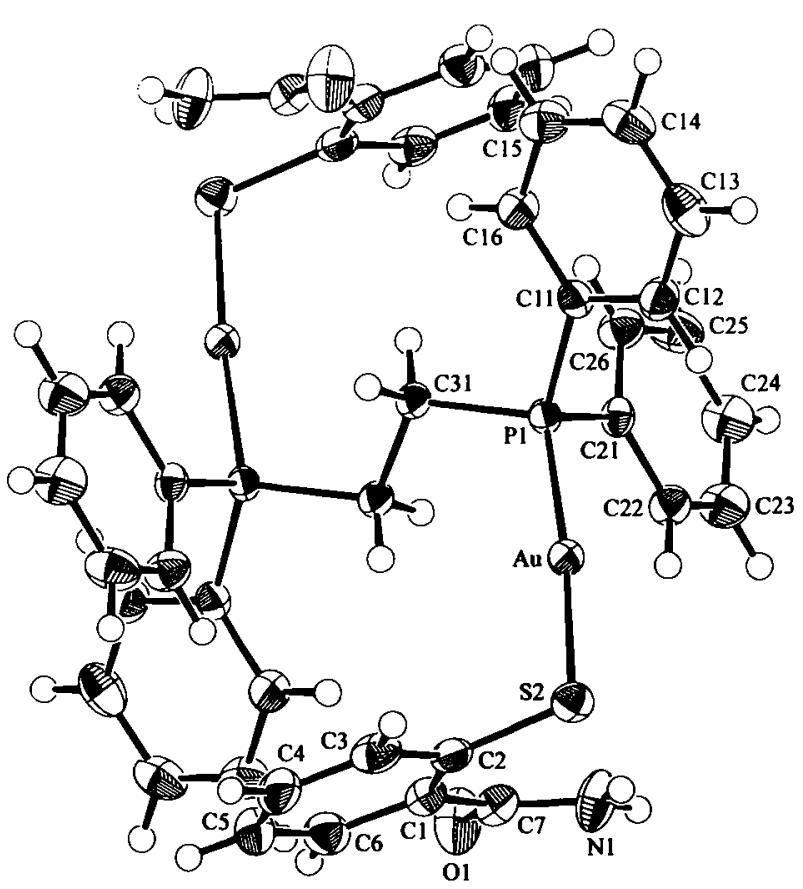

Abstract

$\mathrm{C}_{40} \mathrm{H}_{36} \mathrm{Au}_{2} \mathrm{~N}_{2} \mathrm{O}_{2} \mathrm{P}_{2} \mathrm{~S}_{2}$, triclinic, $P \overline{1}$ (No. 2), $a=10.673(2) \AA$,

$b=11.078(2) \AA, c=9.066(2) \AA, \alpha=107.54(2)^{\circ}, \beta=113.58(2)^{\circ}$, $\gamma=84.84(2)^{\circ}, V=936.2 \AA^{3}, Z=1, R_{\mathrm{gt}}(F)=0.026$,

$w R_{\text {ref }}\left(F^{2}\right)=0.065, T=173 \mathrm{~K}$

\section{Source of material}

The title compound was prepared in $82 \%$ yield from the $1: 2$ reaction between $\left(\mathrm{Ph}_{2} \mathrm{PCH}_{2} \mathrm{CH}_{2} \mathrm{PPh}_{2}\right)(\mathrm{AuCl})_{2}$ and 2-mercaptobenzamide [1]. Pale-yellow crystals were obtained from the vapour diffusion of acetone into a dimethylformamide solution of the compound; mp $513 \mathrm{~K}-515 \mathrm{~K}$ (decomposition).

\section{Experimental details}

The $H$ atoms were placed in their geometrically calculated positions and included in the final refinement in the riding model approximation.

\section{Discussion}

The dinuclear molecule is situated about a crystallographic centre of inversion so that the thiolate groups are anti. The gold atom exists in a linear environment, $174.92(4)^{\circ}$, defined by $\mathrm{S}(d(\mathrm{Au}-\mathrm{S})$ $=2.316(1) \AA)$ and $\mathrm{P}(d(\mathrm{Au}-\mathrm{P})=2.251(1) \AA)$ donors. Molecules aggregate via hydrogen bonding interactions involving the amide groups to form chains along the $a$-direction. Thus, $d\left(\mathrm{H}_{1} \mathrm{a} \cdots \mathrm{Ol}^{i}\right)$ is $2.03 \AA, d\left(\mathrm{N1} \cdots \mathrm{O}^{i}\right)$ is $2.883(7) \AA$ and the angle at $\mathrm{H} 1 \mathrm{a}$ is $162^{\circ}$ for symmetry operation $i:-x, 1-y, 1-z$. The second amide hydrogen atom, $\mathrm{H} 1 \mathrm{~b}$, forms an intramolecular interaction with the $\mathrm{S}$ atom so that $d(\mathrm{H} 1 \mathrm{~b} \cdots \mathrm{S} 2)$ is $2.33 \AA, d(\mathrm{~N} 1 \cdots \mathrm{S} 2)$ is $3.046(5) \AA$ and the angle at $\mathrm{Hlb}$ is $138^{\circ}$. Chains are linked by $\mathrm{Au} \cdots \mathrm{S}^{i i}$ interactions (3.403(2) $\AA$ ) formed between centrosymmetrically molecules resulting in the formation of ( $\mathrm{Au}$...S) rectangles; symmetry operation ii: $-x, 1-y,-z$. The structure of the title compound is isomorphous with the carboxylic acid analogue [2].

Table 1. Data collection and handling.

\begin{tabular}{ll}
\hline Crystal: & $\begin{array}{l}\text { pale-yellow block, } \\
\text { size } 0.06 \times 0.13 \times 0.21 \mathrm{~mm}\end{array}$ \\
& Mo $K_{a}$ radiation $(0.71069 \AA)$ \\
Wavelength: & $80.89 \mathrm{~cm}^{-1}$ \\
$\mu:$ & Rigaku AFC7R, $\omega / 2 \theta$ \\
Diffractometer, scan mode: & $55^{\circ}$ \\
$2 \theta_{\text {max }}:$ & 4524,4291 \\
$N(h k l)_{\text {measured }}, N(h k l)_{\text {unique: }}:$ & $I_{\text {obs }}>2 \sigma\left(l_{\text {obs }}\right), 3477$ \\
Criterion for $I_{\text {obs }}, N(h k l)_{g t}:$ & 226 \\
$N(\text { param })_{\text {refined: }}$ & teXsan [3], DIRDIF92 [4], SHELXI-97 [5], \\
Programs: & DIFABS [6], ORTEPI [7]
\end{tabular}

Table 2. Atomic coordinates and displacement parameters (in $\AA^{2}$ ).

\begin{tabular}{llrrrr}
\hline Atom & Site & \multicolumn{1}{c}{$\boldsymbol{x}$} & \multicolumn{1}{l}{$\boldsymbol{y}$} & \multicolumn{1}{c}{$z$} & \multicolumn{1}{c}{$U_{\text {iso }}$} \\
\hline $\mathrm{H}(1 \mathrm{a})$ & $2 i$ & -0.0679 & 0.4773 & $\mathbf{0 . 3 4 1 6}$ & 0.051 \\
$\mathrm{H}(1 \mathrm{~b})$ & $2 i$ & -0.0607 & 0.4006 & 0.1747 & 0.051 \\
$\mathrm{H}(3)$ & $2 i$ & 0.1390 & 0.0804 & -0.0943 & 0.042 \\
$\mathrm{H}(4)$ & $2 i$ & 0.2188 & -0.0788 & 0.0332 & 0.050 \\
$\mathrm{H}(5)$ & $2 i$ & 0.2276 & -0.0488 & 0.3060 & 0.048 \\
$\mathrm{H}(6)$ & $2 i$ & 0.1498 & 0.1352 & 0.4390 & 0.043 \\
$\mathrm{H}(12)$ & $2 i$ & 0.2785 & 0.6749 & 0.0129 & 0.039 \\
$\mathrm{H}(13)$ & $2 i$ & 0.3667 & 0.7449 & -0.1468 & 0.053 \\
$\mathrm{H}(14)$ & $2 i$ & 0.6017 & 0.7677 & -0.0610 & 0.046 \\
$\mathrm{H}(15)$ & $2 i$ & 0.7493 & 0.7154 & 0.1781 & 0.043 \\
$\mathrm{H}(16)$ & $2 i$ & 0.6642 & 0.6490 & 0.3401 & 0.035 \\
$\mathrm{H}(22)$ & $2 i$ & 0.1595 & 0.6834 & 0.4036 & 0.036 \\
$\mathrm{H}(23)$ & $2 i$ & 0.1165 & 0.8607 & 0.5917 & 0.046 \\
$\mathrm{H}(24)$ & $2 i$ & 0.2796 & 1.0233 & 0.7501 & 0.048 \\
$\mathrm{H}(25)$ & $2 i$ & 0.4915 & 1.0096 & 0.7304 & 0.049 \\
$\mathrm{H}(26)$ & $2 i$ & 0.5371 & 0.8330 & 0.5410 & 0.040 \\
$\mathrm{H}(31 \mathrm{a})$ & $2 i$ & 0.5638 & 0.4589 & 0.3799 & 0.030 \\
$\mathrm{H}(31 \mathrm{~b})$ & $2 i$ & 0.6011 & 0.5912 & 0.5257 & 0.030 \\
& & & & & \\
\hline
\end{tabular}

\footnotetext{
* Correspondence author (e-mail: chmtert@nus.edu.sg)

1 Current address: National University of Singapore,

Department of Chemistry, Singapore 117543
} 
Table 3. Atomic coordinates and displacement parameters (in $\AA^{2}$ ).

\begin{tabular}{|c|c|c|c|c|c|c|c|c|c|c|}
\hline Atom & Site & $x$ & $y$ & $z$ & $U_{11}$ & $U_{22}$ & $U_{33}$ & $U_{12}$ & $U_{13}$ & $U_{23}$ \\
\hline $\mathbf{A u}$ & $2 i$ & $0.20603(2)$ & $0.46782(2)$ & $0.14226(2)$ & $0.0232(1)$ & $0.02330(9)$ & $0.02429(9)$ & $0.00083(6)$ & $0.00903(7)$ & $0.00690(6)$ \\
\hline$S(2)$ & $2 i$ & $0.0320(1)$ & $0.3158(1)$ & $-0.0271(2)$ & $0.0279(6)$ & $0.0304(6)$ & $0.0266(6)$ & $-0.0032(5)$ & $0.0071(5)$ & $0.0059(5)$ \\
\hline $\mathbf{P}(1)$ & $2 i$ & $0.3877(1)$ & $0.6014(1)$ & $0.3106(2)$ & $0.0219(6)$ & $0.0207(5)$ & $0.0227(6)$ & $0.0028(4)$ & $0.0093(5)$ & $0.0083(4)$ \\
\hline$O(1)$ & $2 i$ & $0.0860(5)$ & $0.3488(4)$ & $0.5103(5)$ & $0.061(3)$ & $0.054(3)$ & $0.034(2)$ & $0.021(2)$ & $0.021(2)$ & $0.012(2)$ \\
\hline$N(1)$ & $2 i$ & $-0.0361(5)$ & $0.4131(4)$ & $0.2833(6)$ & $0.042(3)$ & $0.047(3)$ & $0.031(2)$ & $0.021(2)$ & $0.015(2)$ & $0.008(2)$ \\
\hline$C(1)$ & $2 i$ & $0.0961(5)$ & $0.2205(5)$ & $0.2543(6)$ & $0.024(2)$ & $0.030(2)$ & $0.030(2)$ & $-0.000(2)$ & $0.011(2)$ & $0.005(2)$ \\
\hline$C(3)$ & $2 i$ & $0.1401(5)$ & $0.0916(5)$ & $0.0143(7)$ & $0.034(3)$ & $0.036(3)$ & $0.041(3)$ & $0.001(2)$ & $0.025(2)$ & $0.003(2)$ \\
\hline $\mathrm{C}(4)$ & $2 i$ & $0.1885(6)$ & $-0.0025(5)$ & $0.0896(8)$ & $0.034(3)$ & $0.031(3)$ & $0.062(4)$ & $0.008(2)$ & $0.024(3)$ & $0.010(3)$ \\
\hline$C(5)$ & $2 i$ & $0.1928(6)$ & $0.0150(5)$ & $0.2511(8)$ & $0.037(3)$ & $0.032(3)$ & $0.052(3)$ & $0.006(2)$ & $0.015(3)$ & $0.016(2)$ \\
\hline$C(6)$ & $2 i$ & $0.1469(5)$ & $0.1245(5)$ & $0.3297(7)$ & $0.034(3)$ & $0.039(3)$ & $0.035(3)$ & $-0.003(2)$ & $0.011(2)$ & $0.012(2)$ \\
\hline $\mathrm{C}(7)$ & $2 i$ & $0.0480(5)$ & $0.3359(5)$ & $0.3582(6)$ & $0.026(3)$ & $0.033(3)$ & $0.031(3)$ & $0.000(2)$ & $0.012(2)$ & $0.004(2)$ \\
\hline$C(11)$ & $2 i$ & $0.4619(5)$ & $0.6556(4)$ & $0.1937(6)$ & $0.026(2)$ & $0.021(2)$ & $0.025(2)$ & $0.001(2)$ & $0.010(2)$ & $0.007(2)$ \\
\hline$C(12)$ & $2 i$ & $0.3746(5)$ & $0.6839(5)$ & $0.0480(6)$ & $0.028(3)$ & $0.038(3)$ & $0.032(3)$ & $0.005(2)$ & $0.011(2)$ & $0.014(2)$ \\
\hline $\mathrm{C}(13)$ & $2 i$ & $0.4270(7)$ & $0.7254(5)$ & $-0.0473(7)$ & $0.060(4)$ & $0.043(3)$ & $0.034(3)$ & $0.003(3)$ & $0.018(3)$ & $0.021(2)$ \\
\hline$C(15)$ & $2 i$ & $0.6533(6)$ & $0.7083(5)$ & $0.1454(7)$ & $0.038(3)$ & $0.036(3)$ & $0.036(3)$ & $0.000(2)$ & $0.022(2)$ & $0.004(2)$ \\
\hline$C(16)$ & $2 i$ & $0.6027(5)$ & $0.6683(5)$ & $0.2409(6)$ & $0.029(3)$ & $0.035(3)$ & $0.025(2)$ & $0.002(2)$ & $0.012(2)$ & $0.009(2)$ \\
\hline $\mathrm{C}(21)$ & $2 i$ & $0.3530(5)$ & $0.7406(4)$ & $0.4554(5)$ & $0.025(2)$ & $0.025(2)$ & $0.021(2)$ & $0.005(2)$ & $0.010(2)$ & $0.008(2)$ \\
\hline $\mathrm{C}(22)$ & $2 i$ & $0.2273(5)$ & $0.7494(5)$ & $0.4698(6)$ & $0.028(3)$ & $0.030(2)$ & $0.030(3)$ & $0.000(2)$ & $0.012(2)$ & $0.006(2)$ \\
\hline $\mathrm{C}(23)$ & $2 i$ & $0.2019(6)$ & $0.8547(5)$ & $0.5807(7)$ & $0.044(3)$ & $0.037(3)$ & $0.043(3)$ & $0.004(2)$ & $0.030(3)$ & $0.008(2)$ \\
\hline$C(24)$ & $2 i$ & $0.2988(6)$ & $0.9507(5)$ & $0.6752(7)$ & $0.051(4)$ & $0.035(3)$ & $0.040(3)$ & $0.004(2)$ & $0.032(3)$ & $0.000(2)$ \\
\hline$C(25)$ & $2 i$ & $0.4242(6)$ & $0.9432(5)$ & $0.6629(7)$ & $0.051(4)$ & $0.031(3)$ & $0.043(3)$ & $-0.008(2)$ & $0.025(3)$ & $0.001(2)$ \\
\hline$C(26)$ & $2 i$ & $0.4516(5)$ & $0.8382(5)$ & $0.5516(6)$ & $0.031(3)$ & $0.032(3)$ & $0.035(3)$ & $-0.001(2)$ & $0.016(2)$ & $0.004(2)$ \\
\hline$C(31)$ & $2 i$ & $0.5265(5)$ & $0.5275(4)$ & $0.4484(6)$ & $0.023(2)$ & $0.029(2)$ & $0.028(2)$ & $0.005(2)$ & $0.013(2)$ & $0.013(2)$ \\
\hline
\end{tabular}

Acknowledgments. The Australian Research Council and The University of Adelaide are thanked for support.

\section{References}

1. Smyth, D. R.: Structural studies of phosphinegold(I) thiolates with potential medicinal applications. $\mathrm{Pb}$. $\mathrm{D}$. Thesis. The University of Adelaide, 2001.

2. Smyth, D. R.; Vincent, B. R.; Tiekink, E. R. T.: Solvent mediated disruption of intermolecular association in phosphinegold(I) thiolates: the relative importance of carboxylic acid dimer formation on crystal structure. CrystEngComm (2000) Art. No. 21 (6 pages).

3. teXsan: Single Crystal Structure Analysis Software. Version 1.05. Molecular Structure Corporation. The Woodlands, TX, USA 1997.
4. Beurskens, P. T.; Admiraal, G.; Beurskens, G.; Bosman, W. P.; GarcíaGranda, S.; Smits, J. M. M.; Smykalla, C.: The DIRDIF program system, Technical Report of the Crystallography Laboratory, University of Nijmegen, The Netherlands 1992.

5. Sheldrick, G. M.: SHELXL-97. Program for crystal structure refinement. University of Göttingen, Germany 1997.

6. Walker, N.; Stuart, D.: An empirical method for corrrecting diffractometer data for absorption effects. Acta Crystallogr. A39 (1983) 158-166.

7. Johnson, C. K.: ORTEPII. Report ORNL-5138, Oak Ridge National Laboratory, Tennessee, USA 1976. 